\title{
Actitudes parentales hacia la crianza y perfil de socialización en niños escolarizados de Sincelejo
}

\section{Parental Attitudes towards Parenting and Socialization Profile in schooled children in Sincelejo}

\author{
María Laura Vergara-Álvarez. \\ Corporación Universitaria del Caribe CECAR. Facultad de Humanidades y Educación, Programa de Psicología. \\ $\triangle$ maria.vergaraa@cecar.edu.co \\ (iD) https://orcid.org/0000-0001-9600-2956 \\ Liliana Margarita Meza-Cueto. \\ Corporación Universitaria del Caribe - CECAR. Facultad de Humanidades y Educación, Programa de Psicología. \\ $\triangle$ liliana.mezac@cecar.edu.co \\ (iD) https://orcid.org/0000-0003-0860-7512
}

\author{
Nancy Rocío Peña-Oviedo. \\ Corporación Universitaria del Caribe CECAR. Facultad de Humanidades y Educación, Programa de Psicología. \\ $\triangle$ nancy.pena@cecar.edu.co \\ iD https://orcid.org/0000-0003-1926-9838
}

Lilibeth Galindo-Solórzano.

Corporación Universitaria del Caribe CECAR.

Facultad de Humanidades y Educación, Programa de Psicología. Recibido: 07-02-2019

$\triangle$ lilibeth.galindo@cecar.edu.co

(iD) https://orcid.org/0000-0003-2493-6084

Recibido: $07-02-2019$
Aceptado: $27-05-2019$

Publicado: 29-05-2019

\section{RESUMEN}

El objetivo de la presente investigación fue establecer la relación entre las actitudes parentales hacia la crianza y perfil de socialización en niños y niñas de primer y segundo grado de la Institución educativa rural la Peñata en el municipio de Sincelejo. Se realizó bajo una metodología con paradigma positivista, de enfoque cuantitativo, tipo correlacional, diseño no experimental y de corte transversal, en el cual se aplicó el Cuestionario de Crianza Parental PCRI-M y la Batería de Socialización BAS 1. La muestra estuvo constituida por 57 madres con un rango de edad de 22 a 63 años y 57 niños seleccionados de manera intencionada cuyas edades oscilaban entre 6 y13 años con una media de edad de 9,1 años, donde el $49,12 \%$ niñas y $50,8 \%$ niños. El 59,6\% de los estudiantes se encontraba cursando primer grado y el $40,4 \%$ segundo grado. El análisis de los datos se realizó por medio de los estadísticos descriptivos empleando el programa SPSS y la fórmula R-Pearson para el análisis correlacional. En los resultados obtenidos se evidenció que, la actitud hacia la crianza con la media más alta fue el compromiso. En cuanto al perfil de socialización se obtuvo que la dimensión con la media más alta fue el respeto-autocontrol lo que indica que la mayoría de niños acatan normas de convivencia y son capaces de relacionarse armónicamente con sus compañeros. Finalmente, se obtuvo como resultado la no existencia de correlaciones significativas entre las variables, lo cual podría ser explicado a partir de múltiples agentes y factores contextuales e individuales.

Palabras clave: crianza, socialización, correlación, padres, infancia. 


\section{ABSTRACT}

The objective of this research was to establish the relationship between parental attitudes towards upbringing and socialization profile in first and second grade boys and girls of the rural educational institution La Peñata in the municipality of Sincelejo. It was carried out under a methodology with a positivist paradigm, quantitative approach, correlational type, non-experimental design and cross-sectional, in which the Parental Parenting Questionnaire PCRI-M and the BAS 1 Socialization Battery were applied. The sample consisted of 57 mothers with an age range of 22 to 63 years and 57 intentionally selected children whose ages ranged from 6 to 13 years with an average age of 9.1 years, where $49.12 \%$ girls and $50.8 \%$ boys. $59.6 \%$ of the students were in first grade and $40.4 \%$ were in second grade. Data analysis was carried out using descriptive statistics using the SPSS programme and the r-pearson formula for correlational analysis. In the results obtained, it was evident that the attitude towards parenting with the highest average was commitment. Regarding the socialization profile, it was found that the dimension with the highest average was respect-self-control, which indicates that most children abide by the rules of coexistence and are able to relate harmoniously with their peers. Finally, the result was that there were no significant correlations between the variables, which could be explained by multiple agents and contextual and individual factors.

Keywords: upbringing, socialization, correlation, parents, childhood.

\section{INTRODUCCIÓN}

Las formas de violencia y las agresiones entre niños y niñas que se encuentran en edad escolar, se ha transformado en un fenómeno social que causa preocupación a los docentes y a los padres de familia quienes intentan continuamente que las instituciones de educación sean entornos de formación integral, pero la preocupación incrementa cuando las conductas violentas se vuelven constantes y toman distintas maneras de manifestación en sus hijos, ocasionando múltiples problemáticas relacionadas con el establecimiento del perfil de socialización y la convivencia en general (González, Mariaca y Arias, 2014).

Ahora, vale la pena resaltar que las actitudes parentales hacia la crianza son las cogniciones que predisponen al cuidador para que actué de forma positiva o negativa hacia el niño e involucran la aceptación o el rechazo, la frialdad o la calidez que se pueda presentar en la relación entre padres, madres, cuidadores e hijos (Grusec \& Danyliuk, 2014) y la Socialización es un proceso de influencia, intercambio e interacción entre el niño, el grupo social y el contexto en el que nace y crece, por medio del cual lleva a cabo la satisfacción de necesidades y se apropia de la cultura (Gispert, 2001, p. 146), es en este sentido donde se hace necesario revisar detalladamente la asociación que puede existir entre estas variables, soportadas en el modelo bidimensional de Maccoby \& Martins, los cuales mencionan dos dimensiones relacionadas con el proceso de crianza, la primera de estas: la comunicación-el afecto y la segunda: las dimensiones de exigencia-control; y el modelo bidimensional de Kohn quien plantea dos factores dentro del proceso de socialización, entre ellos, los problemas de conducta y los problemas de personalidad (Lacunza, Caballero y Contini, 2013), lo cuales en la actualidad son conocidos como problemas internalizantes y externalizantes.

En este sentido, González, Mariaca y Arias (2014), señalaron que el $60 \%$ de los niños que se encuentran en edad escolar han utilizado la violencia con sus pares en la escuela 
Vergara-Álvarez et al - Actitudes parentales hacia la crianza y perfil de socialización en niños escolarizado.

al menos una vez. Algunos infantes experimentan sentimientos de culpa ante la emisión de la conducta violenta, mientras que otros, experimentan satisfacción ante la emisión del acto, lo que da cuenta, que, aunque es inadecuado agredir, en cierta medida está permitiendo la descarga de emociones y puede producir satisfacción en los niños, convirtiéndose esto en una problemática de orden psicosocial, debido a que tiende a normalizar las conductas violentas en este tipo de poblaciones.

Herrera-Lozano, Vergara-Álvarez y Meza-Cueto (2018), resaltaron en una investigación realizada en una zona rural del municipio de Sincelejo, que el $89,7 \%$ de los niños que se encuentran en edad escolar ha sido reconocido como víctima por parte de sus compañeros de clase, lo cual sugiere niveles alarmantes de victimización, agresividad e intolerancia en estudiantes de básica primaria.

Así mismo, según el investigador Chaux (2003), en Colombia 1 de cada 5 niños es víctima de matoneo en alguna de sus diferentes manifestaciones, donde se evidencia que las cifras más elevadas se encuentran en regiones impactadas por el conflicto armado y la violencia. En el departamento de Sucre, se registró que el $75 \%$ de los estudiantes ha presenciado casos de maltrato entre iguales, y un $100 \%$ de los niños han sido espectadores de algún caso de maltrato, Bullying o intimidación escolar (González, Tamara y Guerra, 2016).

En otro sentido, se debe tener en cuenta el rol que cumplen los padres en el hogar, ya que puede que no se encuentren al pendiente de sus hijos, no establezcan límites claros en el hogar o incluso que exista maltrato intrafamiliar, lo que podría generar la réplica de estos comportamientos en los diferentes escenarios donde se desenvuelven socialmente, derivándose, sintomatología internalizante y externalizante, además de problemáticas relacionadas con la inseguridad, la ansiedad, actos de violencia, la venganza, bajo rendimiento escolar, deserción y depresión en las victimas de esta situación problema (Contreras, 2013).

Cabrera, Guevara y Barrera (2006), expresan que la crianza permisiva, conlleva a la aparicion de cierta sintomatología internalizante y externalizante en el individuo, entendiendo el componente externalizante o pertubador del proceso de socialización como el comportamiento externo que afecta a otros, incluyendo conductas como: el rompimiento de normas con ausencia de sentimiento de culpabilidad y la agresión que conduce a alternaciones en el control de comportamiento, que no son adapatativos y pueden ser desafiantes y antisociales. Ahora, se debe resaltar la importancia que tiene el rol que cumple la familia como el pilar afectivo más sólido en la vida de todo ser humano, decisivo en el proceso de socialización, que propicia el desarrollo integral de sus miembros y en donde los padres cumplen funciones esenciales para el desarrollo psicosocial y comportamental de los menores (Sarmiento, 2000).

Es aquí, donde Franco y Pérez (2014) mencionan que los padres que ejercen poca disciplina observan mayores dificultades y problemas de atención, sueño, conductas agresivas, menores habilidades sociales, depresión, retraimiento e hiperactividad en los niños. Al igual que cuando los padres proporcionan poco afecto, se evidencia en los niños problemas de atención, atipicidad, hiperactividad y menores habilidades sociales. De modo similar, destacaron que cuando los cuidadores están poco comprometidos con la crianza, los hijos tienden a presentar mayores conductas agresivas, hiperactividad, reactividad emocional y pocas habilidades sociales.

De este modo, el liderazgo y la autorregulación juega un papel fundamental en la socialización, ya que representa una dimensión facilitadora del proceso y genera gran 
impacto en la vida individual, grupal y social, debido a que influye significativamente en como los individuos hacen frente a la vida cotidiana y resuelven sus problemas (Mawson, 2011). Ahora, el estilo de crianza que utilizan los padres afecta de forma implícita el establecimiento del perfil de socialización del niño, lo que a su vez genera implicaciones en la predicción del liderazgo. Además de que la formación en creencias, intereses, tradiciones, ocupaciones y nociones de modernidad ayuda a la conformación de habilidades y valores que influyen en la aparición y práctica de la jovilidad, el respeto, el autocontrol y de otros factores relacionados (Wade \& Tavris, 2008).

Carpintero, Castillo, Sibaja y Romero-Acosta (2015) realizaron una investigación con el objetivo de conocer la relación existente entre las variables de interés para esta investigación. Como resultado obtuvieron que la actitud hacia la crianza más prevalente fue el compromiso con la crianza y que la deseabilidad social de los padres, se relacionaría con elevados niveles de ansiedad en los infantes. En este sentido, padres muy preocupados por el rol de sus hijos a nivel social y por parecer buenos padres, podrían influir en la aparición de conductas ansiosas en sus hijos.

Ahora bien, la niñez se encuentra sumida en diversas problemáticas como abuso sexual, explotación infantil, infancia vinculada a la prostitución, niños y niñas vinculados al conflicto armado, infancia afectada por la ola invernal, entre otros, lo que evidencia que se está ante una población vulnerable y que realmente se encuentra en riesgo psicosocial. Así, Salazar, Angarita, Perico, Ruby y Zuluaga, (2011) mencionan que el conflicto armado generó impactos en el sistema familiar, provocando una vulneración directa de los derechos de los niños forjando en estas dificultades para ajustarse socialmente en los diferentes escenarios. Otras de las consecuencias generadas, son el fomento de sentimientos de rabia, temor y vacío emocional, provocando rupturas en los vínculos afectivos y obstaculizando la estructuración de ambientes de crianza y desarrollo en el sistema familiar, fundamentales para los niños y las niñas; por el contrario, dentro del círculo familiar se van configurando las representaciones del conflicto interno y la guerra (Laverde, Joya, castillo y Rodríguez, 2016).

Por todos los motivos mencionados, el presente estudio pretendió responder a la pregunta de investigación: ¿Cuál es la relación entre las actitudes parentales hacia la crianza y el perfil de socialización en niños y niñas de primer y segundo grado de la Institución Educativa Rural la Peñata en el municipio de Sincelejo?

En coherencia a lo anterior, se hace necesario abordar las variables de este estudio, como lo mencionan Muñoz y Molina (2010), quienes consideran que la socialización y otros elementos y comportamientos relacionados, contribuyen a la transformación de las situaciones sociales, pues actúan como instrumentos y mecanismos efectivos, puesto que influyen dentro de las relaciones interpersonales y los grupos sociales, lo cual constituye las bases para las relaciones en sociedad y la conformación de una cultura de paz. Bases que a su vez fomentar y fortalecen la política nacional, que mediante la ley 1620 de 2013 busca generar espacios y estrategias para fortalecer la convivencia escolar y, además, promover la formación en cultura de paz dentro de las instituciones de educación respaldada por el decreto 1038 del año 2015.

De esta manera, en coherencia a la pregunta problema que sitúa la presente investigación tuvo como objetivo principal, establecer la relación entre las Actitudes Parentales hacia la Crianza y el Perfil de Socialización en niños y niñas de primer y segundo grado de la Institución Educativa Rural la Peñata en el municipio de Sincelejo. 
Vergara-Álvarez et al - Actitudes parentales hacia la crianza y perfil de socialización en niños escolarizado.

\section{MÉTODO}

La investigación fue de enfoque cuantitativo, con un tipo de estudio correlacional, cuya "finalidad es identificar el grado de relación o de asociación que pueda existir entre variables de interés dentro de un contexto especifico" (Hernández, Fernández \& Batista, 2010 , p. 85). Se evalúa de manera independiente cada una de las variables dentro de la investigación para luego cuantificar y analizar la relación que puede existir entre ellas. El diseño usado fue no experimental, de tipo transversal, ya que la recolección de datos se realizó en un tiempo y momento único.

Participantes. La población estuvo constituida 57 niños de primer y segundo grado y 57 madres de familia. En cuanto a la población de niños, el $49.12 \%$ fueron niñas y el 50,8\% niños, y sus rangos de edad oscilaban entre los 6 y 13 años, con una media de edad de 9,1 años. El mayor porcentaje correspondió a los estudiantes de primer grado con un $59,6 \%$ y el $40,4 \%$ a segundo grado de básica primaria. Asimismo, las madres de familia, se encontraban entre los 22 y los 65 años, donde el 63,4\% tenían un rango de edad entre 22 y 35 años, el 26,6\% entre 36 y 49 años y el $10 \%$ entre 50 y 62 años de edad, además poseían un nivel educativo básico (primario) en su mayoría, pertenecientes a un estrato socioeconómico bajo. Para la selección de la Muestra Poblacional se empleó un muestreo por conveniencia, el cual consistió en seleccionar de forma puntual un número de sujetos pertenecientes a esta institución y que cumplieran con los siguientes criterios de inclusión: en primer lugar, que cursaran los grados primero y segundo de básica primaria. Se tomaron en cuenta los niños que estuviesen asistiendo a clases durante las fechas de aplicación del instrumento y fueron incluidos los niños cuyos padres dieron su autorización mediante la firma del consentimiento informado.

Instrumentos. Para la variable de Actitudes hacia la Crianza se utilizó el cuestionario de Crianza Parental PCRI-M (Gerard, 1994). Este instrumento permitió conocer las actitudes que predisponen a los padres en el proceso de crianza de sus hijos, esta prueba fue aplicada a las madres de familia. El cuestionario constó de 78 ítems, los cuales están distribuidos en 8 escalas que se muestran a continuación, evidenciando entre paréntesis el alfa de Cronbach obtenido por cada una: Apoyo $(0,711)$, satisfacción con la crianza $(0,816)$, compromiso $(0,800)$, deseabilidad social $(0,723)$, comunicación $(0,757)$, autonomía $(0,754)$, distribución de rol $(0,721)$ y disciplina $(0,763)$. La puntuación por ítem es de 4 puntos que va desde muy de acuerdo a total desacuerdo (Becerra, Roldán y Aguirre, 2008). Para esta investigación se obtuvo un Alpha de Cronbach de 0,768.

Para medir la variable Perfil de Socialización se usó la Batería de Socialización BAS 1 aplicable a docentes (Silva \& Martorell, 1982). Esta prueba permitió conocer el perfil de socialización en los niños a partir de 4 escalas facilitadoras entre estos se encuentran, Jovialidad $(0,924)$, liderazgo $(0,943)$, Respeto-autocontrol $(0,910)$ y Sensibilidad social $(0,900)$, y tres escalas de aspectos inhibidores o perturbadores, las cuales se muestran a continuación seguidas, cada una, del alfa de cronbach arrojado: Apatía-retraimiento $(0,967)$, Ansiedad-timidez $(0,841)$, Agresividad-terquedad $(0,955)$. El instrumento se aplicó a docentes de los grados primero y segundo, quienes dieron cuenta de las conductas sociales de sus estudiantes. También se resalta que por medio de la aplicación de este instrumento se alcanza una apreciación general del grado de ajuste social que tenga el niño. Este instrumento obtuvo un Alpha de Cronbach general de 0,900 .

Procedimiento. Tomando en cuenta los principios éticos, en primera instancia se realizó la firma del consentimiento informado por parte de los padres de familia de los niños y niñas de los grados primero y segundo de la institución educativa rural la Peñata, posterior a esto se realizó una prueba piloto con madres con características poblacionales 
similares a las de la muestra poblacional, con el fin de comprobar que el lenguaje utilizado fuese comprensible para el grupo seleccionado y así justificar la factibilidad del instrumento. A partir de esto, la prueba fue aplicada a la muestra poblacional de madres de familia y posteriormente a los docentes de los niños participantes de la investigación para dar cuenta de la conducta social. Finalmente se diligenció la información en una base de datos y se hicieron los análisis respectivos en el programa SPSS, utilizando los estadísticos descriptivos, empleando la media poblacional y en cuanto al componente correlacional, se utilizó la fórmula estadística R-Pearson.

\section{RESULTADOS}

Los resultados que se muestran a continuación fueron recolectados para determinar la relación entre las actitudes parentales hacia la crianza y el perfil de socialización en niños y niñas de primer y segundo grado de la Institución educativa rural la Peñata, en el municipio de Sincelejo. Se presentan, inicialmente, mediante tablas de frecuencias para las variables estudiadas: actitudes de los padres hacia la crianza y el perfil de socialización de los niños. Seguido, se muestra detalladamente el análisis descriptivo para cada una de ellas destacando aquellas subescalas con la media más alta y baja. Finalmente, se procede a establecer las correlaciones entre las dimensiones correspondientes.

En los resultados se evidencia que la actitud con la media más alta corresponde al compromiso con la crianza con una media de 47,58 (Tabla 1).

Tabla 1. Actitudes parentales hacia la crianza.

\begin{tabular}{|c|c|c|c|c|}
\hline & Mínimo & Máximo & Media & Desviación Estándar \\
\hline Apoyo & 16 & 69 & 26,14 & 7,062 \\
\hline Satisfacción con la crianza & 56 & 37 & 26,16 & 4,177 \\
\hline Compromiso & 35 & 63 & 47,58 & 5,244 \\
\hline Deseabilidad social & 5 & 17 & 13,28 & 2,631 \\
\hline Comunicación & 17 & 32 & 25,72 & 3,098 \\
\hline Autonomía & 19 & 37 & 30,32 & 4,067 \\
\hline Distribución de rol & 13 & 28 & 20,47 & 3,376 \\
\hline Disciplina & 20 & 37 & 27,56 & 4,243 \\
\hline
\end{tabular}

Fuente: elaboración propia

Tal como se destaca en la tabla 2, en cuanto a la variable de Perfil de Socialización, resultó que la dimensión con media más alta fue respeto-autocontrol con una media de 44,95, seguido por liderazgo y en menor medida ansiedad timidez con una media de 20,72 .

Tabla 2. Perfil de Socialización.

\begin{tabular}{ccccc}
\hline & Mínimo & Máximo & Media & Desviación Estándar \\
\hline Liderazgo & 19 & 63 & 44,14 & 11,746 \\
Sensibilidad Social & 15 & 51 & 35,21 & 7,585 \\
Jovialidad & 21 & 48 & 40,70 & 6,620 \\
Respeto Autocontrol & $\mathbf{2 1}$ & $\mathbf{6 8}$ & $\mathbf{4 4 , 9 5}$ & $\mathbf{9 , 6 3 6}$ \\
Agresividad Terquedad & 17 & 61 & 29,95 & 12,836 \\
Apatía Retraimiento & 19 & 76 & 23,28 & 8,797 \\
Ansiedad Timidez & 13 & 48 & 20,72 & 5,650 \\
\hline
\end{tabular}

Fuente: elaboración propia 
Vergara-Álvarez et al - Actitudes parentales hacia la crianza y perfil de socialización en niños escolarizado.

En cuanto al grado de relación o de asociación entre las variables de interés, se encontró que no existe correlación significativa para ninguna de las dimensiones en el nivel 0,01 (bilateral) ni para el nivel 0,05 (bilateral) (Tabla 3).

Tabla 3. Correlación entre actitudes parentales hacia la crianza y perfil de socialización.

\begin{tabular}{|c|c|c|c|c|c|c|c|c|}
\hline Actitud Perfil & Compromiso & Disciplina & $\begin{array}{c}\text { Satisfacción } \\
\text { Crianza }\end{array}$ & Apoyo & Autonomía & Comunicación & $\begin{array}{l}\text { Deseabilidad } \\
\text { Social }\end{array}$ & $\begin{array}{l}\text { Distribución } \\
\text { de Roles }\end{array}$ \\
\hline Liderazgo & ,070 &,- 174 & ,029 & ,028 &,- 090 &,- 041 & 035 &,- 005 \\
\hline Sensibilidad Social & 113 & ,038 & 139 & ,032 & ,020 &,- 087 & 253 &,- 044 \\
\hline Jovialidad & ,029 &,- 108 &,- 072 &,- 101 &,- 061 & 0,99 &, 061 &,- 020 \\
\hline Respeto Autocontrol &,- 045 &,- 111 & 149 &,- 012 &,- 141 & ,089 &,- 128 & ,092 \\
\hline Agresividad Terquedad & 037 & 158 &,- 058 &,- 058 & 106 &,- 036 &,- 149 & 124 \\
\hline Apatía Retraimiento & 143 &,- 055 & 061 & 072 & ,090 &,- 097 & ,027 &,- 163 \\
\hline Ansiedad Timidez & 191 &,- 091 & ,057 & ,076 & ,079 & -.088 & 013 &,- 126 \\
\hline
\end{tabular}

Fuente: elaboración propia

\section{DISCUSIÓN}

Esta investigación propuso establecer la relación entre las Actitudes parentales hacia la Crianza y el Perfil de Socialización de niños de primer y segundo grado de básica primaria. De los datos obtenidos correspondientes a las actitudes parentales hacia la crianza, se encontró que la actitud más prevalente en los padres de esta muestra poblacional es el compromiso, lo cual indica que los padres se encuentran comprometidos con el proceso de crianza de sus hijos, lo que puede en cierta medida fortalecer la comunicación entre estos e incluso les da a los padres la posibilidad de estar en mayor contacto con ellos y de conocer sus intimidades e inquietudes. De modo similar, Carpintero, Castillo y Sibaja (2015), encontraron que dentro de esta misma región la actitud parental hacia la crianza más prevalente fue el compromiso con la crianza; se debe resaltar que este estudio fue realizado en la ciudad de Sincelejo.

Este hallazgo, también es coherente con los datos obtenidos por Roa y Del Barrio (2001), quienes encontraron que las madres españolas alcanzaron puntuaciones elevadas en la escala de compromiso, satisfacción con la crianza, comunicación y distribución de rol, a diferencia de las madres estadounidenses quienes obtuvieron su máxima puntuación en las dimensiones de autonomía, apoyo, disciplina y deseabilidad social. En concordancia con estos hallazgos, diversos estudios resaltan la importancia que tiene la presencia de los padres en las manifestaciones empáticas, de autocontrol y de sensibilidad moral en los niños, las cuales se encuentran influenciadas significativamente por el grado de compromiso del padre en el proceso de formación y de crianza (Fundación Chile Unido, 2002).

Ahora bien, los resultados del perfil de socialización de los niños evidencian que la dimensión más prevalente fue el respeto-autocontrol, seguido por el liderazgo y en menor medida se presentó la dimensión de ansiedad- timidez, lo que indica que los niños son capaces de cumplir las normas de convivencia y de relacionarse de forma armónica con sus compañeros y las demás personas. Asimismo, para la dimensión de liderazgo, se denota que estos niños tienen altos índices de popularidad, iniciativa y son proactivos en el marco de las relaciones interpersonales. Estos resultados guardan relación con lo expuesto por Montoya y Chávez (2016), quienes encontraron que en sujetos de 13 a 15 años las dimensiones con la incidencia más alta fueron la dimensión de respeto autocontrol y liderazgo y las más bajas estuvieron centradas en ansiedad timidez y retraimiento social 
Los resultados también están en línea con los expuesto por Garaigordobil \& García (2006), quienes resaltan que los niños con autocontrol y liderazgo, suelen ser empáticos, con escasas conductas negativas a nivel social, niveles bajos de agresividad, conducta antisocial, impulsividad y retraimiento. Sin embargo, Lacunza, Caballero y Contini, (2013) demostraron que las condiciones externas y las situaciones de pobreza influyen negativamente en las familias y en los niños, niñas y adolescentes, situación que no va en coherencia a los resultados arrojados por este estudio, debido a que a pesar de que la población participante pertenece a un estatus socioeconómico bajo, se encontró en los niños niveles altos de liderazgo, ya que este grupo tiene un rol protagónico, puesto que poseen ascendencia entre sus pares e iniciativa.

Por otro lado, en cuanto a la correlación de la variable actitudes parentales hacia la crianza y el perfil de socialización, se encontró que no existe una correlación significativa entre ambas variables, resultado que no es consistente con lo expuesto por Zolten \& Long (1997), quienes mencionan que existe una correlación positiva entre la comunicación en el hogar y la dimensión de respeto- autocontrol.

Las diferencias de los resultados del presente estudio con las investigaciones presentadas anteriormente y que evidencian correlación significativa entre las actitudes parentales hacia la crianza y el perfil de socialización, podría explicarse a partir de varios factores. En primer lugar, tomando en cuenta la confluencia de las variables estudiadas en esta investigación, las cuales en la actualidad implican un concepto de influencia paterna que está más lleno de complejidad, puesto que cede a la visión del padre simplemente como un moldeador, y se encarga de integrar elementos que con frecuencia son omitidos en los estudios sobre el proceso de crianza, tales como el propio papel del niño, los roles de los demás contextos y la influencia del componente no familiar (Collins, 2000).

Ahora bien, se hace necesario reconocer la función socializadora de la crianza dentro de los procesos del infante, ya que la vinculación de la crianza al seno de las condiciones culturales y contextuales le da un sentido de especificidad que explicaría la no existencia de correlaciones significativas entre estas variables, entendiendo el establecimiento del perfil de socialización como el resultado de múltiples procesos en los que el niño se encuentra inmerso desde el nacimiento. De acuerdo con esto, Maccoby y Martin (1983) considera que el proceso de socialización es bidireccional, en la cual el niño configura su forma de actuar, sus pensamientos y emociones de acuerdo con las estructuras sociales y culturales que le son transmitidas en el intercambio con su medio y no solamente por las actitudes o los estilos que los padres asumen dentro del proceso de crianza.

Esta concepción, en la cual se considera la existencia de una influencia reciproca tanto dentro del proceso de crianza como dentro del proceso de socialización, producida por una bidireccionalidad entre padres/cuidadores e hijos, evidencia empíricamente el papel activo del infante que socializa. De modo similar Collins (2000), sustenta con diversos estudios la idea de la conexión que existe entre la crianza y otros procesos e influencias, fundamentado en la perspectiva teórica ecológica. Es importante tener en cuenta la influencia de otros contextos, factores y agentes que producen tensión y contribuyen a la sinergia de todo el proceso, tomando en cuenta, que se está ante variables multifacéticas, donde los padres y la familia son apenas un solo elemento en el arduo camino de la socialización. Dentro de estos agentes y factores interrelacionados, tiene gran relevancia la industria, el sistema normativo y jurídico, la cultura, la política, la dimensión social, la económica y el contexto ecológico en general en que se desenvuelve el infante (Amar, Abello y Tirado, 2004). 
Vergara-Álvarez et al - Actitudes parentales hacia la crianza y perfil de socialización en niños escolarizado.

Finalmente, existen otros elementos que pueden explicar el proceso de socialización y el establecimiento del perfil de socialización en el infante como: la percepción del niño, la motivación y la personalidad, estando esta última representada en un constructo que se hace evidente por medio de la conducta social (Celener, 1996). Así, esta variable se encuentra ligada a elementos cognitivos, volitivos, afectivos y motivacionales que son el resultado de una constante interacción entre factores biológicos, históricos, sociales y culturales (Marín, 2009).

\section{CONCLUSIONES}

Las actitudes hacia la crianza son consideradas por diversos autores promotoras del desarrollo de habilidades sociales y adaptativas. En este sentido, Roa \& Del Barrio, (2001) consideran que el compromiso en el proceso de crianza es favorecedor del proceso de socialización del niño. Entre las dimensiones evaluadas en las madres de familia, el compromiso fue más prevalente que otras actitudes, mientras que el respetoautocontrol es la dimensión del perfil de socialización más prevalente para esta muestra poblacional. Sin embargo, en esta población particular no se encontró correlación entre las actitudes parentales y el perfil de socialización de los niños. Se debe mencionar que el proceso de socialización se encuentra influenciado por múltiples factores y agentes de socialización que han tomado fuerza y protagonismo debido a las modificaciones actuales que ha sufrido la familia y que han dotado de mayor influencia dentro del proceso a la escuela, al sector educativo y al componente cultural y social, además de que el propio papel del niño, la personalidad y los otros contextos de desarrollo influyen considerablemente en el establecimiento del perfil de socialización, ya que se trata de una variable multifacética, lo cual puede explicar la no existencia de correlación entre las variables (Collins, 2000).

Los resultados obtenidos no le restan importancia al estudio, por el contrario, se sustrae que no existe correlación significativa entre las variables debido a que tanto el proceso de crianza como el proceso de socialización son situaciones bidireccionales en el niño, donde este tiene un papel activo y recibe influencia de muchos factores asociados, más aun tomando en cuenta la situación social actual, donde las normativas, las leyes, la modificación del sistema familiar y otros componentes contextuales e individuales cumplen un papel fundamental en el establecimiento del perfil de socialización (Jurado, 2004).

Por otro lado, la muestra poblacional del presente estudio estuvo conformada en su totalidad por madres de familia y no hay población de padres, lo que sugeriría un estudio más exhaustivo con una muestra más representativa y homogénea con relación al género. También de cara a posteriores estudios sería interesante analizar la implicación de la variable de género, comparando las actitudes hacia la crianza entre padres y madres y como estas actitudes influyen en los niños y niñas que se encuentran en edad escolar.

Finalmente, es fundamental fomentar el interés por este tipo de estudio en el país y en los psicólogos con formación educativa y familiar, ahondando en estos campos para el fortalecimiento de las políticas públicas, aportando a la transformación de la realidad social y generando un ambiente funcional con la presencia de los padres en los distintos contextos del infante. 


\section{REFERENCIAS}

Amar, J., Abello, R. y Tirado, D. (2004). Desarrollo Infantil y Construcción del Mundo Social. Barranquilla. Barranquilla: Ediciones Universidad del Norte. Recuperado de $\quad$ http://www.scielo.org.co/scieloOrg/php/reflinks.php?refpid=S0120$\underline{5552200700020000400012 \& \operatorname{lng}=p t \& p i d=S 0120-55522007000200004}$

Becerra, S., \& Roldán, W., \& Aguirre, M. (2008). Adaptación del cuestionario de crianza parental (pcri-m) en Canto Grande. Pensamiento Psicológico, 4(11), 135-149. Recuperado de http://www.redalyc.org/html/801/80111671009/

Cabrera, V., Guevara, I., \& Barrera, F. (2006). Relaciones maritales, relaciones paternas y su influencia en el ajuste psicológico de los hijos. Acta Colombiana de Psicología, 9(3), 115-126.

Carpintero, L., Castillo, S., Sibaja, D. y Romero-Acosta, K. (2015). Estilos de crianza y su relación con sintomatología internalizante en estudiantes de 8 a 16 años. Revista De Psicología GEPU, 6(2), 53-65. Retrieved from https://search.proquest.com/docv iew $/ 1950390661$ ?accountid $=34487$

Celener, G. (1996). Fundamentos teóricos para la inclusión de láminas en blanco (OrtTat). Publicación interna de la Cátedra de Técnicas psicodiagnósticas II. (Tesis de pregrado). Buenos Aires: Universidad de Buenos Aires, Facultad de Psicología. Recuperado de http://www.espaciologopedico.com/recursos/glosariodet.php? $\mathrm{Id}=233$

Chaux, E. (2003). Agresión reactiva, agresión instrumental y el ciclo de la violencia. Revista de estudios sociales, Universidad de los Andes, 47-58.

Collins, A (2000) Contemporary Research on Parenting: The case for Nature and Nurture. American Psychologist, 55(2). Recuperado de https://www.researchgate.net/profile/ Willard_Collins/publication/12597856_Contemporary_research_on_parenting_ The_case_for_nature_and_nurture_American_Psychologist_55 2 _218-232/ links/54ad $\overline{8} 1980$ cf24aca1c6fб̄0d8/Contemporary-research-on-parenting-The-casefor-nature-and-nurture-American-Psychologist-552-218-232.pdf

Congreso de la República. (15 de Marzo de 2013) Convivencia escolar. [Ley 1620 de 2013]. DO: 48.733.

Contreras, P. (2013). El fenómeno de Bullying en Colombia. Revista Logos Ciencia \& Tecnología, 4(2), 100-114.

Franco, N., Pérez, M.A. (2014) Relación entre los estilos de crianza parental y el desarrollo de ansiedad y conductas disruptivas en niños de 3 a 6 años. Revista de Psicología Clínica con Niños y Adolescentes, 1(2); 149-156.

Fundación Chile Unido. (2002). El rol del padre y su influencia en los hijos. Corriente de opinión: 64. Recuperado dehttps://www.recercat.cat/bitstream/handle/2072/13775/ TFC-JUANOLA-2009\%282\%29.pdf?sequence $=2$. 
Vergara-Álvarez et al - Actitudes parentales hacia la crianza y perfil de socialización en niños escolarizado.

Garaigordobil, M. (2014). Conducta prosocial: el papel de la cultura, la familia, la escuela y la personalidad. Revista Mexicana de investigación en Psicología, 6(2). Pp.146157. Recuperado de http://www.sc.ehu.es/ptwgalam/art completo/2014/C\%20 Prosocial\%20RMIP\%202014.pdf.

Gerard, A. (1994). Parent-Child Relationship Inventory: Manual. Los Angeles: Westem Psychological Services. Recuperado de https://www.worldcat.org/title/parent-childrelationship-inventory-pcri-manual/oclc/33255596

Gispert, Carlos. (2001). Psicología del niño y del Adolescente. Barcelona, España: Oceano Grupo Editorial, S.A

González, M. Á., Tamara, A., y Guerra, I. D. C. (2016). Perfil del acoso escolar en la institución educativa la unión de Sincelejo, 2014-2015.

González, V., Mariaca, J. I. y Arias J. L. (2014). Estudio exploratorio del bullying en Medellín. Pensando Psicología, 10(17), 17-25. Doi: http://dx.doi.org/10.16925/ pe.v10i17.776

Grusec JE, Danyliuk T. (2014). Actitudes y Creencias Parentales: Su Impacto en el Desarrollo de los Niños. En: Tremblay RE, Boivin M, Peters RDeV, eds. Tremblay RE, ed. tema. Enciclopedia sobre el Desarrollo de la Primera Infancia [en línea]. http:// www.enciclopedia-infantes.com/habilidades-parentales/segun-los-expertos/ actitudes-y-creencias-parentales-su-impacto-en-el.

Hernandez, R., Fernández, C., \& Baptista, P. (2010). Metodología de la investigación. México: McGraw Hill. Recuperado de https://www.esup.edu.pe/descargas/dep investigacion/Metodologia\%20de\%20la\%20investigaci\%C3\%B3n\%205ta\%20 Edici\%C3\%B3n.pdf

Herrera-Lozano, J., Vergara-Álvarez, M., \& Meza-Cueto, L. (2018). Conductas y experiencias sociales en clase de niños escolarizados en el municipio de Sincelejo, Colombia. Búsqueda, 5(21), 212-230. DOI: https://doi.org/10.21892/01239813.423

Izzedin, R. y Pachajoa, A. (2009). Pautas, prácticas y creencias acerca de crianza... ayer y hoy liberabit. Revista de psicología, 15(2), 109-115. Recuperado de: http://www. redalyc.org/pdf/686/68611924005.pdf

Jurado, J. (2004). Socialización Familiar Urbana en Medellín. Problemas y Tendencias Contemporáneas. Revista Historia Crítica. Universidad de los Andes. Recuperado de http://aprendeenlinea.udea.edu.co/Ims/moodle/pluginfile.php/104903/mod resource/content/1/P.A. \%20Teor\%C3\%ADa\%20y\%20Procesos\%20con\% 20 individuo\%20y\%20familia\%20II.pdf

Lacunza, A., \& Caballero, S., \& Contini, E. (2013). Adaptación y evaluación de las propiedades psicométricas de la BAS-3 para población adolescente de Tucumán (Argentina). Diversitas: Perspectivas en Psicología, 9(1), 29-44.

Laverde, L. J., Muñoz Joya, D. J., Osuna Castillo, M., \& Ovalle Rodríguez, L. C. (2016). Representaciones Sociales Sobre el Conflicto Armado en los Niños y Niñas de la Estrategia Atrapasueños de la Secretaría Distrital de Integración Social (Doctoral dissertation, Tesis) Bogotá, Colombia, p. 116. Recuperado de: http://biblioteca. clacso.edu.ar/Colombia/alianza-cinde-umz/20170810123449/RevistaLatinoamerica naVol.15N.2julio-diciembre2017.pdf 
Maccoby, E., \& Martin, J. (1983). Socialization in the context of the family: Parent-child interaction. Handbook of child psychology: Vol. 4. Socialization, personality, and social development, New York: Wiley. Recuperado de https://pdfs.semanticscholar. org/353b/824813759e7330e71281e2dd660604884244.pdf

Marín, J. (2009). Conductas prosociales en el barrio Los Pinos de la ciudad de Barranquilla, Colombia Prosocial behavior in los Pinos neighborhood in Barranquilla city, Colombia. Revista CES Psicología, 2(2), 60-75. Recuperado de https://www.redalyc.org/ pdf/4235/423539413005.pdf

Mawson, B. (2011). Children's leadership strategies in early childhood. Journal of Research in Childhood Education, 25(4), 327-338. doi:10.1080/02568543.2011.6 05207

Ministerio de Educación Nacional. (25 de Mayo 2015) Decreto 1038 [Cátedra de paz]. Cátedra de paz [Ley 1732 de 2014] DO: 49522.

Montoya, G., y Chávez, C. (2016). Evaluación de la socialización en adolescentes. (Tesis de maestría). Escuela de psicología educativa, cuenca. Recuperado de http:// dspace.ucuenca.edu.ec/bitstream/123456789/24330/1/Trabajo\%20Final\%20 de\%20Titulaci\%C3\%B3n.pdf

Muñoz, Francisco A. y Molina Rueda, Beatriz. (2010). Una Cultura de Paz compleja y conflictiva. La búsqueda de equilibrios dinámicos. Revista de Paz y Conflictos Disponible en: <http://ucsj.redalyc.org/articulo.oa?id=205016387004> ISSN

Roa Capilla, Luisa, Barrio, Victoria del, Adaptación del Cuestionario de Crianza Parental (PCRI-M) a población española. Revista Latinoamericana de Psicología [en línea] 2001, 33 [Fecha de consulta: 27 de marzo de 2018] Disponible en: <http://www. redalyc.org/articulo.oa?id $=80533307>$ ISSN 0120-0534

Salazar, J. A. A., Angarita, L. F., Restrepo, L. P., Henao, N. R., \& Zuluaga, Y. E. (2011). Desplazamiento Forzado y conflicto armado. Niños y niñas vulnerados en sus derechos humanos. Revista Orbis, (20), 51-78. Recuperado de https://www.redalyc. org/pdf/709/70922149003.pdf

Sarmiento, M. (2000). Psicoprofilaxis Familiar. Colombia: Ediciones USTA.

Silva, F. y Martorell, C. (1982). Batería de Socialización. Valencia: Promolibro. Recuperado de http://www.web.teaediciones.com/Ejemplos/BAS-1-2 extracto-web.pdf

Viveros, F. y Vergara, C (2014) Familia y dinámica familiar: cartilla dirigida a facilitadores para la aplicación de talleres con familias. Recuperado de: http://www.funlam.edu. co/uploads/fondoeditorial/preliminar/2014/Familia-dinamica-familiar.pdf

Wade, C. \& Tavris, (2008). Psicología. (9a edición), Prentice Hall, Upper Saddle River, NJ.

Zolten, M.A., \& Long, N. (1997). La comunicación entre padres e hijos. Departamento de Pediatría, Universidad de Arkansas para Ciencias Médicas. Arte de Scott Snider. Springdale, Arkansas. Recuperado de http://parenting-ed.org/wp-content/themes/ parenting-ed/files/Spanish-Handouts/Early-Childhood-Handouts/General\%20 Parenting\%20Info/Parental\%20Communication.pdf. 